\title{
DÜBLIN
}

Technological University Dublin ARROW@TU Dublin

2009-01-01

\section{Converging Flow Between Coaxial Cones}

O. Hall

University of Exeter

A. D. Gilbert

University of Exeter

C. P. Hills

Dublin Institute of Technology, chris.hills@dit.ie

Follow this and additional works at: https://arrow.tudublin.ie/scschmatart

Part of the Applied Mathematics Commons, Applied Mechanics Commons, Fluid Dynamics Commons, and the Mathematics Commons

\section{Recommended Citation}

Hall, O., Gilbert, A.D. \& C.P. Hills. (2009). Converging Flow Between Coaxial Cones. Fluid Dynamics

Research, vol. 41, no. 1, pg. 25. doi:10.21427/vbk1-hn07

This Article is brought to you for free and open access by the School of Mathematics at ARROW@TU Dublin. It has been accepted for inclusion in Articles by an authorized administrator of ARROW@TU Dublin. For more information, please contact arrow.admin@tudublin.ie, aisling.coyne@tudublin.ie,gerard.connolly@tudublin.ie.

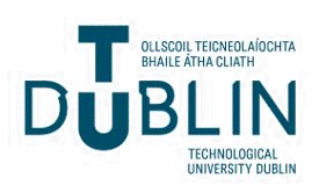




\title{
Converging flow between coaxial cones
}

\author{
Oskar Hall, ${ }^{a}$ Andrew D. Gilbert ${ }^{\mathrm{a}}$ and Christopher P. Hills ${ }^{\mathrm{b}}$ \\ ${ }^{a}$ Mathematics Research Institute, \\ School of Engineering, Computing and Mathematics, \\ University of Exeter, UK. \\ ${ }^{\mathrm{b}}$ School of Mathematical Sciences, \\ Dublin Institute of Technology, Ireland.
}

\begin{abstract}
Fluid flow governed by the Navier-Stokes equation is considered in a domain bounded by two cones with the same axis. In the first, 'non-parallel' case the two cones have the same apex and different angles $\theta=\alpha$ and $\beta$ in spherical polar coordinates $(r, \theta, \phi)$. In the second, 'parallel' case the two cones have the same opening angle $\alpha$, parallel walls separated by a gap $h$ and apices separated by a distance $h / \sin \alpha$. Flows are driven by a source $Q$ at the origin, the apex of the lower cone in the parallel case. The Stokes solution for the non-parallel case is discussed and the angles $(\alpha, \beta)$ identified where it breaks down, analogously to flow in a wedge geometry. For the case of convergent flow, $Q<0$, solutions governed by the Navier-Stokes equation are discussed for both parallel and non-parallel geometries. At large distances the flow is in a viscous regime and takes a Poiseuille profile. As the origin is approached the inertial terms become important and a plug flow emerges, with constant radial velocity in the core, sandwiched between thin boundary layers. By systematic approximation, PDEs are derived that describe the transition from viscous to high Reynolds number flow, and solutions describing the plug flow and boundary layers are obtained using matched asymptotic expansions.
\end{abstract}

Key words: Conical geometry, converging flow, Stokes flow, plug flow, boundary layer.

\section{Introduction}

Fluid flow in a conical geometry has been widely studied, both for flows bounded by a single cone, say the region $\theta \leq \alpha$ in spherical polar coordinates $(r, \theta, \phi)$, or bounded by two cones. In this case there are two natural possibilities, firstly when the cones have the same apex and axis, and so the 
domain may be given by $\alpha \leq \theta \leq \beta$, and secondly when the cones have parallel walls separated by a gap $h$. These two cases are depicted schematically in figure 1 below and we refer to them as the 'non-parallel' and the 'parallel' geometries for brevity. The aim of this paper is the analysis of solutions of the Stokes equation in the non-parallel geometry, and of the Navier-Stokes equation in both geometries, in the case of converging flow.

Applications of flows in these conical geometries include the processing of polymer melts and liquid foods (Ohmura et al., 2004; Noui-Mehidi et al., 1999), centrifugal pumps with medical applications (Nakamura et al., 2001), centrifuges (Bark et al., 1984) and the cone-plane viscometer (Hynes, 1978; Moffatt, 1980). Thus a wide variety of flow problems within conical geometries are of great practical interest, whether driven by sources or sinks, rotating boundaries or combinations of these mechanisms.

One key area of study, in the Stokes regime, has been the possible existence of infinite sequences of Moffatt eddies, tending to the origin in the single cone geometry (Malyuga, 2005; Shankar, 2005) or in the non-parallel two cone geometry (Malhotra et al., 2005). These are typically driven by a far-field disturbance, but may also be driven by weak inertial effects if the boundaries are instead rotated: the leading order flow is pure swirl, but at the order of the Reynolds number, nonlinear terms drive weak meridional flows and possible sequences of eddies (Hynes, 1978; Moffatt, 1980; Hewitt, 2004; Hall et al., 2007). As the Reynolds number is increased the geometry begins to resemble the classical Taylor-Couette experiment particularly for small angle $\alpha$ and a narrow gap (Hoffman \& Busse, 1999; Hewitt, 2004), and experiments in this geometry undertaken by Wimmer (1995), Noui-Mehidi et al. (2002) and Nakamura et al. (2001) show Taylor vortices. For many applications it is natural to introduce sources and sinks. With two cones in the parallel case, Troshkin (1973) considers diverging flow driven by rotating boundaries analytically, and Noui-Mehidi et al. (1999) give numerical solutions when swirling flow is driven by a tangential inlet at the top or bottom of a pair of truncated cones.

In the present paper our interest is in the fundamental case when the boundaries are at rest and a sink or source is introduced at the origin, of strength $Q$. The resulting converging or diverging flows are taken to be steady, axisymmetric, with no swirl component. This problem was considered for the case of a single cone by Bond (1927) and Ackerberg (1965), who suggested that a vortex motion might occur near the apex; this prediction was later discussed critically by Brown \& Stewartson (1965) and has not been observed experimentally. Nonetheless the approach by Ackerberg (1965) has close links with our study as we indicate below.

In the case of converging/diverging flow (with no azimuthal component) be- 

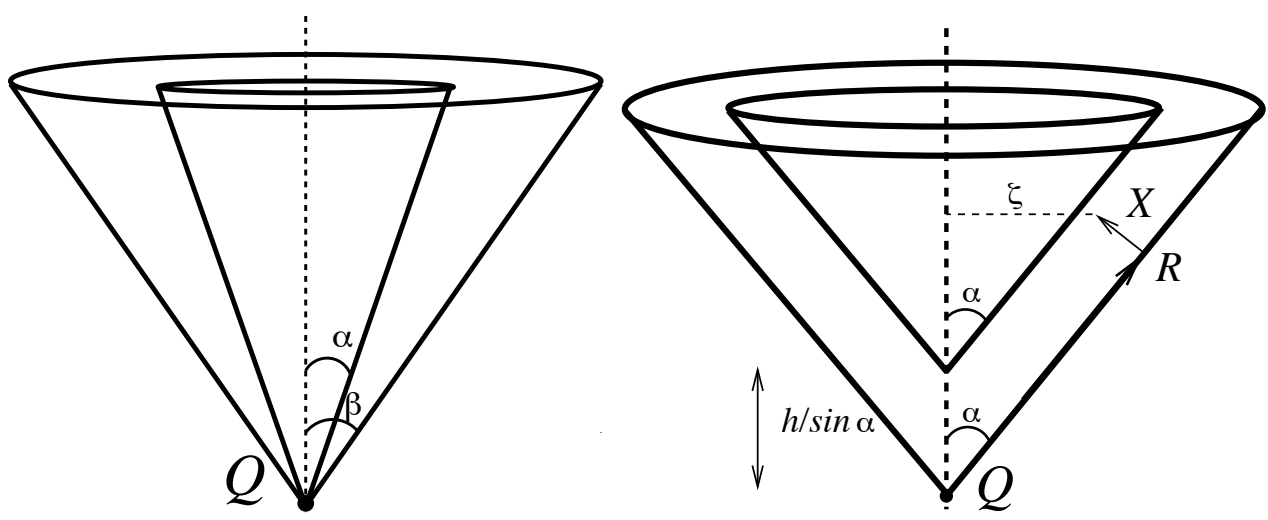

Fig. 1. Depicted are (a) the non-parallel geometry, with origin at the common cone apex, and (b) the parallel geometry, with origin at the apex of the lower cone. In (b) biconical coordinates $(R, \phi, X)$ are depicted.

tween two cones we are only aware of three recent studies. Vatistas \& Ghaly (1999) consider this problem analytically for the non-parallel geometry and develop a system of ODEs describing the profile of the flow, parameterised by the distance from the apex. Here it is important to note that as the flow moves towards the apex between two cones it must accelerate for purely geometrical reasons, and any measure of the Reynolds number increases for both the parallel and non-parallel geometries. In the far field Vatistas \& Ghaly (1999) obtain profiles appropriate to a Stokes regime, but as the apex is approached, the profile takes the form of a plug flow, with uniform radial velocity in the interior, sandwiched between thinning boundary layers. Our work below will confirm many of these general features, in the case of converging flow with a narrow gap; however as Vatistas \& Ghaly (1999) state, their analysis is based on a local linearisation procedure which is somewhat ad hoc and so their results do not constitute a solution of the Navier-Stokes equation. The other two studies, Fohr \& Mallet (1975) and Renaud (1980), focus on the Stokes regime and then bring in the effects of inertia by means of an expansion in inverse powers of distance from the apex (as does Ackerberg (1965) for a single cone). They obtain terms in their expansion, and comment on some singular behaviour for certain cone openings, a topic we will return to in this paper.

It is interesting to compare and contrast briefly the problem of converging flow in the two-cone geometry with the broadly similar example of Jeffery-Hamel flow in an angled corner (see, for example, Fraenkel, 1962; Stow et al., 2001). In this case the geometrical convergence gives a Reynolds number that remains constant as the apex is approached, and so the problem separates exactly in polar coordinates to give an ODE for the profile as a function of angle. In the case of the conical flows we consider this separability property is lost, as viscous terms and inertial terms scale differently. The lack of separability means that a PDE is needed to describe the transition from viscous, Poiseuille flow, to a plug flow at high effective Reynolds number as the apex is approached. 
The present paper is structured as follows. In section 2 we set out the governing equations and in section 3 study Stokes flow in the non-parallel case, with elements of our analysis in common with Renaud (1980), Fohr \& Mallet (1975) and Vatistas \& Ghaly (1999). We find that the solution breaks down for certain cone openings and compare this with the results of the first two of these studies. In section 4 we introduce the effects of inertia but focus on the narrow gap case of the non-parallel geometry, with cone angles $\alpha \simeq \beta$. We derive, using standard asymptotic methods, a PDE that describes the transition from Stokes flow to high Reynolds number flow. This is solved numerically and shows the transition to a plug flow, as found in the study of Vatistas \& Ghaly (1999). The same PDE is obtained by Ackerberg (1965) for flow in a single cone, but not solved numerically. Further asymptotic approximation gives the structure of the boundary layers bounding the plug flow in terms of the solution of a third order ODE (again obtained by Ackerberg (1965)), which we solve numerically. Section 5 gives a similar analysis for the parallel case, and the final section offers a concluding discussion.

\section{Governing equations and geometry}

We consider fluid motion governed by the steady Navier-Stokes equation,

$$
\left(\mathbf{u}^{\prime} \cdot \nabla^{\prime}\right) \mathbf{u}^{\prime}=-\nabla^{\prime} p^{\prime}+\nu \nabla^{\prime 2} \mathbf{u}^{\prime}, \quad \nabla^{\prime} \cdot \mathbf{u}^{\prime}=0
$$

The dashes refer to dimensional variables and we consider the two geometries shown in figure 1. In both cases we have two coaxial cones. For the first case, figure 1(a), the cones have a common apex which is the origin, and are given by $\theta=\alpha$ and $\theta=\beta$ in spherical polar coordinates $(r, \theta, \phi)$. We refer to this as the 'non-parallel case'. For the second case, figure 1(b), the cones have parallel sides separated by a gap $h$ and non-coincident apices a distance $h / \sin \alpha$ apart. The origin is at the lower apex and the lower cone is given by $\theta=\alpha$; this is the 'parallel case'.

For these two geometries we are interested in the axisymmetric, steady flows generated when no-slip boundary conditions are imposed on the cones and a source of strength $Q$ is placed at the origin giving a volume flux of $2 \pi Q$. Much of our analysis is limited to the case when there is a sink, $Q<0$, at the origin, but we leave $Q$ to have either sign. The two cases are somewhat different: in the non-parallel case the geometry is specified by two angles $\alpha$ and $\beta$ which do not introduce a length-scale into the problem. However in the parallel case there is the gap width $h$ which allows us to define a Reynolds number.

Consider first the non-parallel case. We use the two quantities $\nu$ and $\mathcal{Q} \equiv|Q|$ 
to non-dimensionalise the problem with

$$
r^{\prime}=\mathcal{Q} r / \nu, \quad \mathbf{u}^{\prime}=\nu^{2} \mathbf{u} / \mathcal{Q}, \quad p^{\prime}=\nu^{4} p / \mathcal{Q}^{2} .
$$

We then have the following problem to solve for $\mathbf{u}(r, \theta)$,

$$
\begin{aligned}
(\mathbf{u} \cdot \nabla) \mathbf{u} & =-\nabla p+\nabla^{2} \mathbf{u}, \quad \nabla \cdot \mathbf{u}=0 \\
\mathbf{u}(r, \alpha) & =\mathbf{u}(r, \beta)=0, \quad \int_{S} \mathbf{u} \cdot \mathrm{d} \mathbf{S}= \pm 2 \pi .
\end{aligned}
$$

in dimensionless variables, with the \pm referring to the sign of $Q$ and $S$ a surface spanning the conical opening with outwards normal. Although there is no Reynolds number as such in the non-parallel problem, it is useful to define an effective Reynolds number, which is a function of radius. To do this we note that as a function of radius the dimensional quantity $\mathbf{u}^{\prime}$ scales as $u^{\prime}=\mathcal{Q} / r^{\prime 2}$ and so we can set

$$
\operatorname{Re}_{\mathrm{eff}}(r)=r^{\prime} u^{\prime} / \nu=\mathcal{Q} / r^{\prime} \nu=r^{-1}
$$

Thus while there is no single Reynolds number in the problem, with our nondimensionalisation $r \gg 1$ corresponds to a viscous, Stokes regime, whereas $r \ll 1$ gives a large Reynolds number flow.

The parallel problem, depicted in figure 1(b), is different in that we can generate a Reynolds number $R e$ from the dimensional problem using the gap width $h$, with

$$
R e \equiv \varepsilon^{-1}=\mathcal{Q} / h \nu .
$$

We can then non-dimensionalise using (2.2) to obtain the problem for $\mathbf{u}(R, X)$ written as (2.3) again with, now,

$$
\mathbf{u}(R, 0)=\mathbf{u}(R, \varepsilon)=0, \quad \int_{S} \mathbf{u} \cdot \mathrm{d} \mathbf{S}= \pm 2 \pi .
$$

Here $(R, \phi, X)$ is the biconical coordinate system indicated schematically in figure 1(b) and defined below in section 5. Clearly in the parallel problem we cannot say much analytically about the 'apex region' when $r^{\prime}=O(h)$ and there is a transition from motion between two cones, to motion towards the apex of the lower cone. For large $R e$, we will see that again there is a transition from viscous flow at large $r$ to high Reynolds number flow, still between the parallel cones, before reaching the apex region.

\section{Stokes flow in the non-parallel case}

We first consider Stokes flow in the non-parallel geometry for which we omit the inertial terms $\mathbf{u} \cdot \nabla \mathbf{u}$ from the governing equations (2.3), (2.4). This will 
provide the large- $r$ solution to the non-dimensional problem above. We introduce a streamfunction $\psi(r, \theta)$ with

$$
u_{r}=\frac{1}{r^{2} \sin \theta} \frac{\partial \psi}{\partial \theta}, \quad u_{\theta}=-\frac{1}{r \sin \theta} \frac{\partial \psi}{\partial r} .
$$

After a change of variable $t=\cos \theta$, the governing equations for the flow become,

$$
\begin{aligned}
\mathbb{D}^{4} \psi & \equiv \mathbb{D}^{2} \mathbb{D}^{2} \psi \equiv\left[\frac{\partial^{2}}{\partial r^{2}}+\frac{1-t^{2}}{r^{2}} \frac{\partial^{2}}{\partial t^{2}}\right]^{2} \psi=0, \\
\psi(r, \alpha) & =0, \quad \psi(r, \beta)= \pm 1, \quad \frac{\partial \psi}{\partial \theta}(r, \alpha)=0, \quad \frac{\partial \psi}{\partial \theta}(r, \beta)=0 .
\end{aligned}
$$

As in Moffatt \& Duffy (1980) the streamfunction will consist of a particular part $\psi_{\mathrm{p}}$ driven by the source at the apex and a homogeneous part $\psi_{\mathrm{h}}$. It is the homogeneous part arising purely from the geometry that may give an infinite sequence of eddies near the apex or in the far field (Malhotra et al., 2005; Hall et al., 2007). In the far field where $r \gg 1$, the stream function takes the form

$$
\psi=\psi_{\mathrm{p}}+\psi_{\mathrm{h}}= \pm f(\cos \theta)+\sum_{i=1}^{\infty} a_{i} r^{-\lambda_{i}+3} f_{i}(\cos \theta)
$$

where $\lambda_{i}$ are eigenvalues with positive real part, and $f_{i}$ the corresponding eigenfunctions.

We consider only the particular part, which satisfies the following equation,

$$
6 f^{\prime \prime}+\frac{\mathrm{d}^{2}}{\mathrm{~d} t^{2}}\left(\left(1-t^{2}\right) f^{\prime \prime}\right)=0
$$

with $f(\cos \alpha)=0, f(\cos \beta)=1$. Our analysis now is similar to that in Fohr \& Mallet (1975), Renaud (1980) and Vatistas \& Ghaly (1999). The transformation $f^{\prime \prime}(t)=\left(1-t^{2}\right)^{-1 / 2} g(t)$ results in the equation

$$
\left(1-t^{2}\right) g^{\prime \prime}-2 t g^{\prime}+\left(6-\left(1-t^{2}\right)^{-1}\right) g=0,
$$

and it follows that $g(t)$ can be written as a sum of associated Legendre functions $P_{n}^{m}, Q_{n}^{m}$ or Legendre functions $P_{n}, Q_{n}$ as

$$
g(t)=c_{1} P_{2}^{1}(t)+c_{2} Q_{2}^{1}(t)=-\left(1-t^{2}\right)^{1 / 2}\left(c_{1} P_{2}^{\prime}(t)+c_{2} Q_{2}^{\prime}(t)\right)
$$

(Abramowitz \& Stegun, 1965). Writing $g$ in terms of $f^{\prime \prime}$ and integrating gives

$$
f^{\prime}(t)=-c_{1} P_{2}(t)-c_{2} Q_{2}(t)-c_{3}
$$


with $c_{3}$ a constant of integration. We note for reference that

$$
P_{2}(t)=\frac{3 t^{2}-1}{2}, \quad Q_{2}(t)=\frac{3 t^{2}-1}{4} \log \frac{1+t}{1-t}-\frac{3 t}{2} .
$$

Now the radial velocity is $u_{r}=\mp r^{-2} f^{\prime}$ from (3.1), (3.4). Applying the no-slip boundary condition that $f^{\prime}=0$ at $\theta=\alpha, \beta$ yields

$$
f^{\prime}(t)=-c_{1}\left[P_{2}(t)+b_{2} Q_{2}(t)+b_{3}\right]
$$

with

$$
b_{2} \equiv \frac{c_{2}}{c_{1}}=-\frac{P_{2}\left(t_{2}\right)-P_{2}\left(t_{1}\right)}{Q_{2}\left(t_{2}\right)-Q_{2}\left(t_{1}\right)}, \quad b_{3} \equiv \frac{c_{3}}{c_{1}}=\frac{P_{2}\left(t_{2}\right) Q_{2}\left(t_{1}\right)-P_{2}\left(t_{1}\right) Q_{2}\left(t_{2}\right)}{Q_{2}\left(t_{2}\right)-Q_{2}\left(t_{1}\right)},
$$

and we have for brevity set $t_{1} \equiv \cos \alpha, t_{2} \equiv \cos \beta$.

Next we need to apply $f=0$ at $t=t_{1}$ and $f=1$ at $t=t_{2}$. We integrate $f^{\prime}$ and impose these conditions to write $f$ as

$$
f(t)=-c_{1}\left[R_{2}(t)-R_{2}\left(t_{1}\right)+b_{2}\left(S_{2}(t)-S_{2}\left(t_{1}\right)\right)+b_{3}\left(t-t_{1}\right)\right],
$$

where $R_{2}$ and $S_{2}$ are the integrals of $P_{2}$ and $Q_{2}$, explicitly,

$$
R_{2}(t)=\frac{t^{3}-t}{2}, \quad S_{2}(t)=\frac{t^{3}-t}{4} \log \frac{1+t}{1-t}-\frac{t^{2}}{2},
$$

and

$$
c_{1}=-\left[Q_{2}\left(t_{2}\right)-Q_{2}\left(t_{1}\right)\right] / D\left(t_{1}, t_{2}\right) .
$$

The quantity $D$ is

$$
\begin{aligned}
D\left(t_{1}, t_{2}\right) & =\left[Q_{2}\left(t_{2}\right)-Q_{2}\left(t_{1}\right)\right]\left[R_{2}\left(t_{2}\right)-R_{2}\left(t_{1}\right)\right]-\left[P_{2}\left(t_{2}\right)-P_{2}\left(t_{1}\right)\right]\left[S_{2}\left(t_{2}\right)-S_{2}\left(t_{1}\right)\right] \\
& +\left[P_{2}\left(t_{2}\right) Q_{2}\left(t_{1}\right)-P_{2}\left(t_{1}\right) Q_{2}\left(t_{2}\right)\right]\left(t_{2}-t_{1}\right)
\end{aligned}
$$

and after some algebra this may be simplified to give

$$
4 D\left(t_{1}, t_{2}\right)=\left[t_{1}^{3}\left(3 t_{2}^{2}-1\right)-t_{2}^{3}\left(3 t_{1}^{2}-1\right)\right] \log \gamma-6 t_{1} t_{2}\left(t_{2}-t_{1}\right)^{2},
$$

with

$$
\gamma=\frac{\left(1+t_{2}\right)\left(1-t_{1}\right)}{\left(1-t_{2}\right)\left(1+t_{1}\right)} .
$$

This solution is given in an equivalent form in Vatistas \& Ghaly (1999).

With all the coefficients determined, the Stokes flow can be computed for a variety of cone openings $\alpha$ and $\beta$, and figure 2 shows some examples plotted for the case of inflow, that is with the lower sign above. A point that is not noted in previous studies is that the flow becomes undefined for cone openings $\alpha$ 
(a)

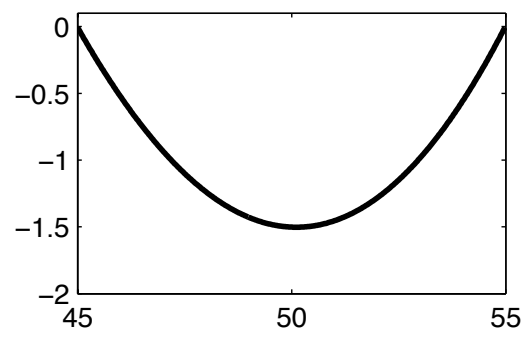

(c)

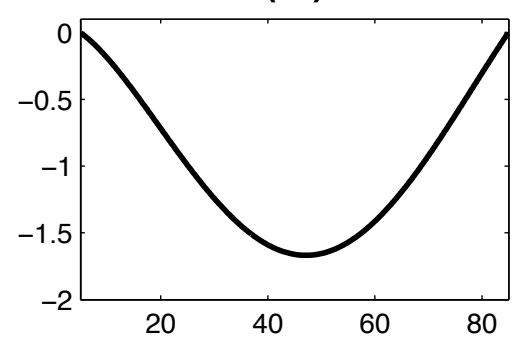

(b)

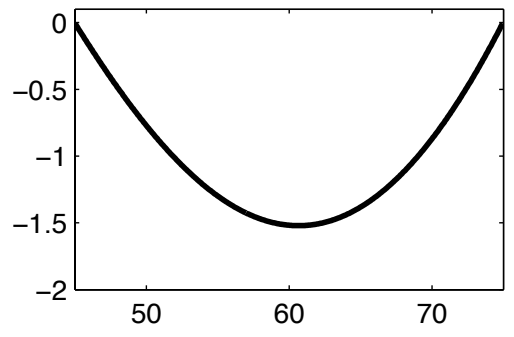

(d)

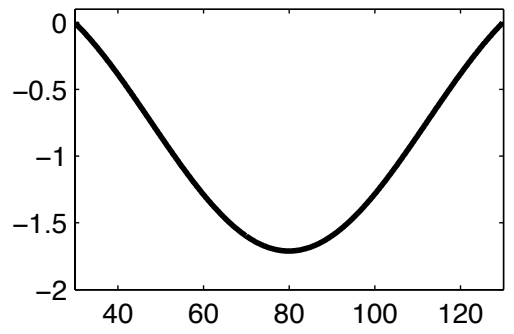

Fig. 2. The scaled radial velocity component $\delta r^{2} u_{r}=\delta f^{\prime}$ in the case of inflow (lower sign), plotted for cone openings with angles (a) $\alpha=45^{\circ}, \beta=55^{\circ}$, (b) $\alpha=45^{\circ}$, $\beta=75^{\circ}$, (c) $\alpha=5^{\circ}, \beta=85^{\circ}$ and (d) $\alpha=30^{\circ}, \beta=130^{\circ}$, where in all the figures the $x$-axis represents the angle.

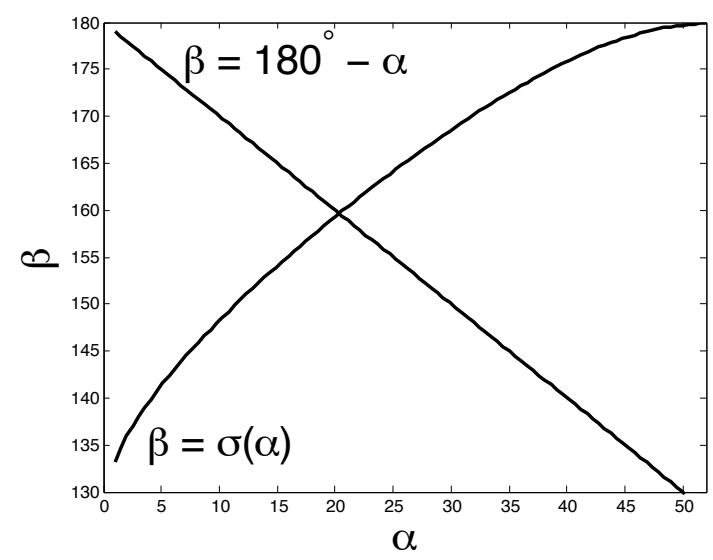

Fig. 3. The curve in the $(\alpha, \beta)$ plane defined by $D\left(t_{1}, t_{2}\right)=0$, for which the particular solution breaks down, together with the cut $\alpha+\beta=180^{\circ}$.

and $\beta$ where $D\left(t_{1}, t_{2}\right)=D(\cos \alpha, \cos \beta)$ vanishes. The cone openings for which this can occur are shown as points on a curve $\beta=\sigma(\alpha)$ in the $(\alpha, \beta)$-plane in figure 3 . It may be seen that we need a wide opening, with moderately small values of $\alpha$ and $\pi-\beta$ for breakdown to occur.

Although breakdown generally occurs for points on the curve $\beta=\sigma(\alpha)$ in 
(a)

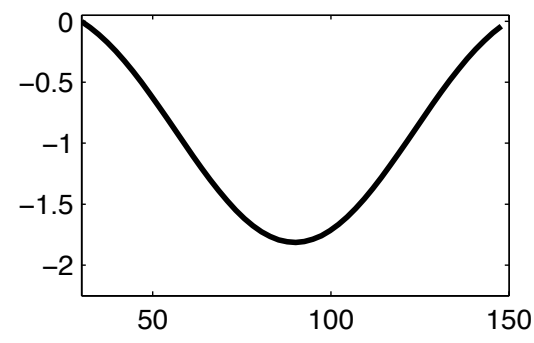

(c)



(b)

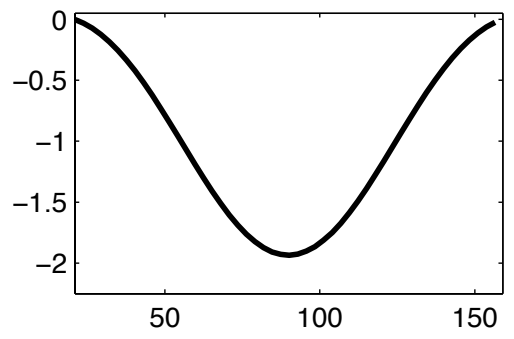

(d)

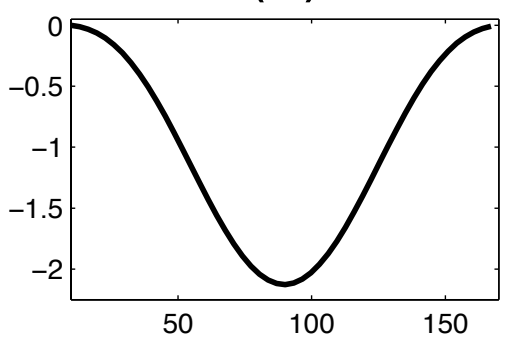

Fig. 4. The scaled radial velocity component $\delta r^{2} u_{r}=\delta f^{\prime}$ plotted for inflow and symmetric cone openings with $\alpha+\beta=180^{\circ}$ and angles (a) $\alpha=30^{\circ}$, (b) $21^{\circ}$, (c) $20^{\circ}$ and $(\mathrm{d}) 10^{\circ}$, where in all the figures the $x$-axis represents the angle.

figure 3, there is one exception, which is the symmetrical opening $\alpha=\pi-\beta$, depicted as a straight line. At the point where the curve and line intersect, although $D\left(t_{1}, t_{2}\right)$ vanishes there, simultaneously the numerator of $c_{1}$ in (3.14) is zero and the singularity is removable. In fact for a symmetrical opening, $t_{2}=-t_{1}$, there are significant simplifications as the radial velocity is even in $t$, with

$$
c_{1}=-\frac{1}{2} t_{1}^{-3}, \quad b_{2}=0, \quad b_{3}=-P_{2}\left(t_{1}\right) .
$$

In this case there is no singular behaviour, for the whole range of $\alpha$.

To illustrate these points, figure 4 shows profiles for inflow in the symmetrical case $\alpha=\pi-\beta$, which remain well-behaved for all values of $\alpha$. On the other hand figure 5 depicts profiles for a slightly asymmetrical case $\alpha=178^{\circ}-\beta$. As the singular curve is approached, the velocity profile develops a region of backflow and the peak values of the velocity increase. Just beyond the singularity, the flow profile flips sign. Analogous singular behaviour has been discussed for Stokes flow in a corner by Moffatt \& Duffy (1980) and this signals that global effects will play a role. In fact the leading exponent $\lambda_{1}$ of the homogenous component of the solution (3.4) passes through 3 at the same point (Hall, 2007); hence the homogeneous solution becomes of the same order of dominance as the particular one and cannot be neglected. (It is interesting to note that Malyuga (2005) finds that for a cone geometry the viscous terms 
(a)

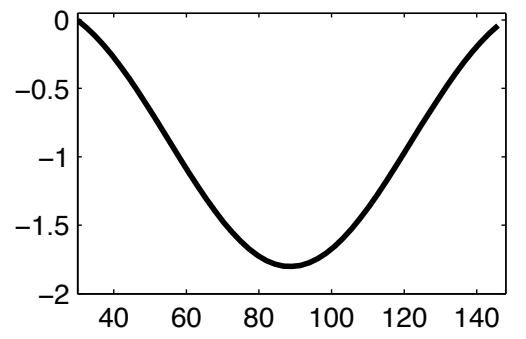

(c)

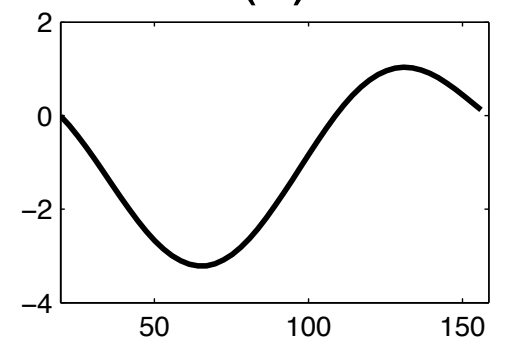

(b)

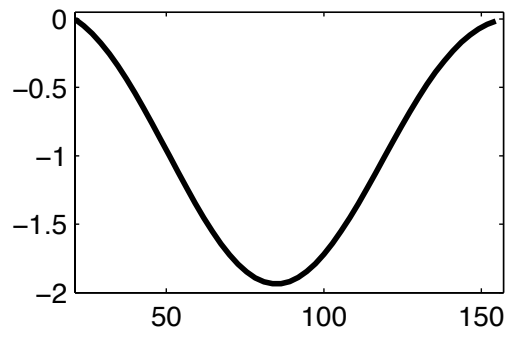

(d)

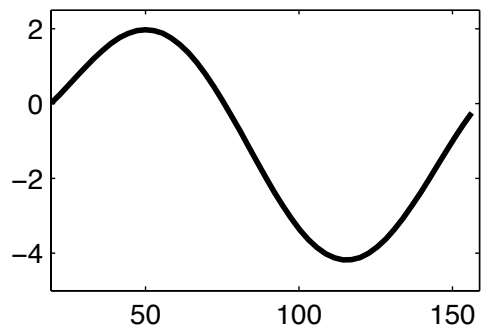

Fig. 5. The scaled radial velocity component $\delta r^{2} u_{r}=\delta f^{\prime}$ plotted for inflow and slightly asymmetric cone openings with $\alpha+\beta=178^{\circ}$ and angles (a) $\alpha=30^{\circ}$, (b) $21^{\circ}$, (c) $19.5^{\circ}$ and (d) $19.25^{\circ}$, where in all the figures the $x$-axis represents the angle.

will be dominant over the inertial for these modes and any cone opening.)

For the conical asymmetric geometry this singular behaviour was not discussed in Vatistas \& Ghaly (1999) and is not present in the leading order solutions obtained by Fohr \& Mallet (1975) and Renaud (1980), as they focus on the symmetric case and on the cone-plane case $0 \leq \alpha \leq \beta=\pi / 2$. We note that they do find singular behaviour in the symmetric case at an angle $\alpha \simeq 12^{\circ}$ in the first correction arising from inertia; this lies beyond the removable singularity $D(\cos \alpha,-\cos \alpha)=0$ at the dot in figure 3 . In the next section we introduce inertia, but in a narrow gap limit; so we are not able to make contact with these results, and leave this for future study. 


\section{Flow in the non-parallel case for a narrow gap}

We now include the inertial terms and consider the system (2.1) which may be written out, for axisymmetric flow, as

$$
\begin{aligned}
& u_{r} \frac{\partial u_{r}}{\partial r}+\frac{u_{\theta}}{r} \frac{\partial u_{r}}{\partial \theta}-\frac{u_{\theta}^{2}}{r}=-\frac{\partial p}{\partial r}+\frac{1}{r^{2}} \frac{\partial}{\partial r}\left(r^{2} \frac{\partial u_{r}}{\partial r}\right)+\frac{1}{r^{2} \sin \theta} \frac{\partial}{\partial \theta}\left(\sin \theta \frac{\partial u_{r}}{\partial \theta}\right) \\
&-\frac{2 u_{r}}{r^{2}}-\frac{2}{r^{2} \sin \theta} \frac{\partial}{\partial \theta}\left(\sin \theta u_{\theta}\right) \\
& u_{r} \frac{\partial u_{\theta}}{\partial r}+\frac{u_{\theta}}{r} \frac{\partial u_{\theta}}{\partial \theta}+\frac{u_{r} u_{\theta}}{r}=-\frac{1}{r} \frac{\partial p}{\partial \theta}+\frac{1}{r^{2}} \frac{\partial}{\partial r}\left(r^{2} \frac{\partial u_{\theta}}{\partial r}\right)+\frac{1}{r^{2} \sin \theta} \frac{\partial}{\partial \theta}\left(\sin \theta \frac{\partial u_{\theta}}{\partial \theta}\right) \\
&+\frac{2}{r^{2}} \frac{\partial u_{r}}{\partial \theta}-\frac{u_{\theta}}{r^{2} \sin ^{2} \theta}, \\
& \frac{1}{r^{2}} \frac{\partial}{\partial r}\left(r^{2} u_{r}\right)+\frac{1}{r \sin \theta} \frac{\partial}{\partial \theta}\left(\sin \theta u_{\theta}\right)=0
\end{aligned}
$$

We focus on the limit of narrow angular gap, setting

$$
\delta=\beta-\alpha \ll 1
$$

and use this as a small parameter in asymptotic expansions. We need to modify the definition of the effective Reynolds number in the limit of small $\delta$ as we have two length scales, the distance $r^{\prime}$ to the apex and the gap width $\delta r^{\prime}$, using dimensional coordinates. The flow magnitude is $u^{\prime}=\mathcal{Q} / \delta r^{\prime 2}$, and it turns out that we should estimate the inertial term $\left(\mathbf{u}^{\prime} \cdot \nabla^{\prime}\right) \mathbf{u}^{\prime}$ as $u^{\prime 2} / r^{\prime}$ but the viscous term $\nu \nabla^{\prime 2} \mathbf{u}^{\prime}$ as $\nu u^{\prime} / \delta^{2} r^{\prime 2}$ to give the ratio as the new effective Reynolds number

$$
\operatorname{Re}_{\mathrm{eff}}(r)=\left(u^{\prime 2} / r^{\prime}\right) /\left(\nu u^{\prime} / \delta^{2} r^{2}\right)=\delta^{2} u^{\prime} r^{\prime} / \nu=\delta \mathcal{Q} / r^{\prime} \nu=\delta r^{-1}
$$

Thus the small Reynolds number regime corresponds to $r \gg \delta$ and the large Reynolds number regime to $r \ll \delta$. We will first investigate Stokes flow in the regime $r=O(1)$ and then move into the transition regime $\operatorname{Re}_{\mathrm{eff}}(r)=O(1)$ when $r=O(\delta)$.

\subsection{Stokes flow and the introduction of inertia}

For $r=O(1)$ and $\delta \ll 1$ a solution to the system (4.1-4.3) can be developed as a regular perturbation series. We set

$$
\theta=\alpha+\delta x, \quad \frac{\partial}{\partial \theta}=\frac{1}{\delta} \frac{\partial}{\partial x} .
$$


and use the stream function defined in (3.1). These quantities are expanded as functions of $x$ and $r$ as

$$
\begin{aligned}
u_{r} & =\delta^{-1} u_{0}+u_{1}+\cdots, \quad u_{\theta}=v_{0}+\delta v_{1}+\cdots, \\
\psi & =\psi_{0}+\delta \psi_{1}+\cdots, \quad p=\delta^{-3} p_{0}+\delta^{-2} p_{1}+\cdots,
\end{aligned}
$$

and substituted into (3.1), (4.1) and (4.2). At leading order (4.2) shows that $p_{0}=p_{0}(r)$, independent of $x$, and the following equations must be satisfied,

$$
0=-\frac{d p_{0}}{d r}+\frac{1}{r^{2}} \frac{\partial^{2} u_{0}}{\partial x^{2}}, \quad u_{0}=\frac{1}{r^{2} \sin \alpha} \frac{\partial \psi_{0}}{\partial x}, \quad v_{0}=-\frac{1}{r \sin \alpha} \frac{\partial \psi_{0}}{\partial r},
$$

with $u_{0}=\psi_{0}=0$ at $x=0$ and $u_{0}=0, \psi_{0}= \pm 1$ on $x=1$. Straightforward calculations then give the leading order solution as Poiseuille flow

$$
u_{0}=\mp \frac{6 x(x-1)}{r^{2} \sin \alpha}, \quad v_{0}=0, \quad \psi_{0}=\mp x^{2}(2 x-3), \quad p_{0}= \pm \frac{4}{r^{3} \sin \alpha} .
$$

At next order again $p_{1}=p_{1}(r)$ and the following equations hold,

$$
\begin{aligned}
& u_{0} \frac{\partial u_{0}}{\partial r}+v_{0} \frac{\partial u_{0}}{\partial x}=-\frac{\mathrm{d} p_{1}}{\mathrm{~d} r}+\frac{1}{r^{2}} \frac{\partial^{2} u_{1}}{\partial x^{2}}+\frac{\cos \alpha}{r^{2} \sin \alpha} \frac{\partial u_{0}}{\partial x}, \\
& u_{1}=\frac{1}{r^{2} \sin \alpha} \frac{\partial \psi_{1}}{\partial x}-\frac{x \cos \alpha}{r^{2} \sin ^{2} \alpha} \frac{\partial \psi_{0}}{\partial x}, \\
& v_{1}=-\frac{1}{r \sin \alpha} \frac{\partial \psi_{1}}{\partial r}+\frac{x \cos \alpha}{r \sin ^{2} \alpha} \frac{\partial \psi_{0}}{\partial r},
\end{aligned}
$$

with $u_{1}=\psi_{1}=0$ at $x=0,1$. These give (Hall, 2007),

$$
\begin{aligned}
& u_{1}= \pm \frac{2 \cos \alpha}{r^{2} \sin ^{2} \alpha}\left(x^{3}-x\right)-\frac{6}{35 r^{3} \sin ^{2} \alpha}\left(14 x^{6}-42 x^{5}+35 x^{4}-9 x^{2}+2 x\right), \\
& v_{1}=-\frac{6}{35 r^{3} \sin ^{2} \alpha}\left(2 x^{7}-7 x^{6}+7 x^{5}-3 x^{3}+x^{2}\right) \\
& \psi_{1}=\mp \frac{\cos \alpha}{\sin \alpha}\left(x^{4}-2 x^{3}+x^{2}\right)-\frac{6}{35 r \sin \alpha}\left(2 x^{7}-7 x^{6}+7 x^{5}-3 x^{3}+x^{2}\right), \\
& p_{1}=\mp \frac{2 \cos \alpha}{r^{3} \sin ^{2} \alpha}-\frac{27}{35 r^{4} \sin ^{2} \alpha} .
\end{aligned}
$$

There are two effects driving the corrections at this order. The first is the last term on the right-hand side of (4.10), which brings in the first effect of the conical geometry. This drives viscous corrections with \pm signs, which are the first of the pairs of terms in $u_{1}, \psi_{1}$ and $p_{1}$ above. It does not drive a flow in $v_{1}$ however, and the powers of $r$ involved do not make the overall expansion (4.7) non-uniform. What we are simply seeing is a perturbation expansion of the Stokes flow between cones, identified in the previous section 3.

Of more interest to us are the second terms in the equations for $u_{1}, \psi_{1}, p_{1}$, and the whole of $v_{1}$. These are driven by the inertial terms on the left-hand side of 
equation (4.10). Comparing the powers of $r$ with those in (4.9), we see that the expansion (4.7) becomes non-uniform when $r=O(\delta)$ : the inertial terms must play a role at this point, as suggested by the increase in the effective Reynolds number (4.5) to values of order unity. These inertial terms are present in the perturbation expansion, in inverse powers of the radial distance $r$, of Fohr \& Mallet (1975) and Renaud (1980) for general cone openings and in Ackerberg (1965) for a single cone.

\subsection{The transition to high Reynolds number flow}

The previous section suggests a rescaling when $r=O(\delta)$ and we set

$$
r=\frac{\delta s}{\sin \alpha}, \quad \frac{\partial}{\partial r}=\frac{\sin \alpha}{\delta} \frac{\partial}{\partial s},
$$

in addition to (4.6). We expand quantities as functions of $x$ and $s$ with

$$
\begin{aligned}
u_{r} & =\sin \alpha\left(\delta^{-3} u_{0}+\delta^{-2} u_{1}+\cdots\right), \quad u_{\theta}=\sin \alpha\left(\delta^{-2} v_{0}+\delta^{-1} v_{1}+\cdots\right) \\
\psi & =\psi_{0}+\delta \psi_{1}+\cdots, \quad p=\sin ^{2} \alpha\left(\delta^{-6} p_{0}+\delta^{-5} p_{1}+\cdots\right)
\end{aligned}
$$

The factors of $\sin \alpha$ here eliminate the parameter $\alpha$ from the problem. These expansions are then substituted into the full equations (3.1), (4.1) and (4.2) and powers of $\delta$ are equated. From (4.2) we obtain at the leading order of $\delta^{-8}$,

$$
0=-\frac{1}{s} \frac{\partial p_{0}}{\partial x}
$$

so that $p_{0}=p_{0}(s)$ is constant across the narrow gap.

At the next order we obtain the key governing PDE from (4.1) which we write, dropping the subscript zero from $u_{0}, v_{0}, p_{0}$ and $\psi_{0}$ to avoid clutter, as

$$
u \frac{\partial u}{\partial s}+\frac{v}{s} \frac{\partial u}{\partial x}=-\frac{\mathrm{d} p}{\mathrm{~d} s}+\frac{1}{s^{2}} \frac{\partial^{2} u}{\partial x^{2}}
$$

(Ackerberg, 1965). Continuity is imposed from (3.1) by requiring that

$$
u=\frac{1}{s^{2}} \frac{\partial \psi}{\partial x}, \quad v=-\frac{1}{s} \frac{\partial \psi}{\partial s}
$$

which results in a single PDE for $\psi$,

$$
\frac{1}{s^{2}} \frac{\partial \psi}{\partial x} \frac{\partial}{\partial s}\left(\frac{1}{s^{2}} \frac{\partial \psi}{\partial x}\right)-\frac{1}{s^{4}} \frac{\partial \psi}{\partial s} \frac{\partial^{2} \psi}{\partial x^{2}}=-\frac{\mathrm{d} p}{\mathrm{~d} s}+\frac{1}{s^{4}} \frac{\partial^{3} \psi}{\partial x^{3}},
$$

and the boundary conditions are given by

$$
\psi(s, 0)=0, \quad \psi(s, 1)= \pm 1, \quad u(s, 0)=u(s, 1)=0 .
$$



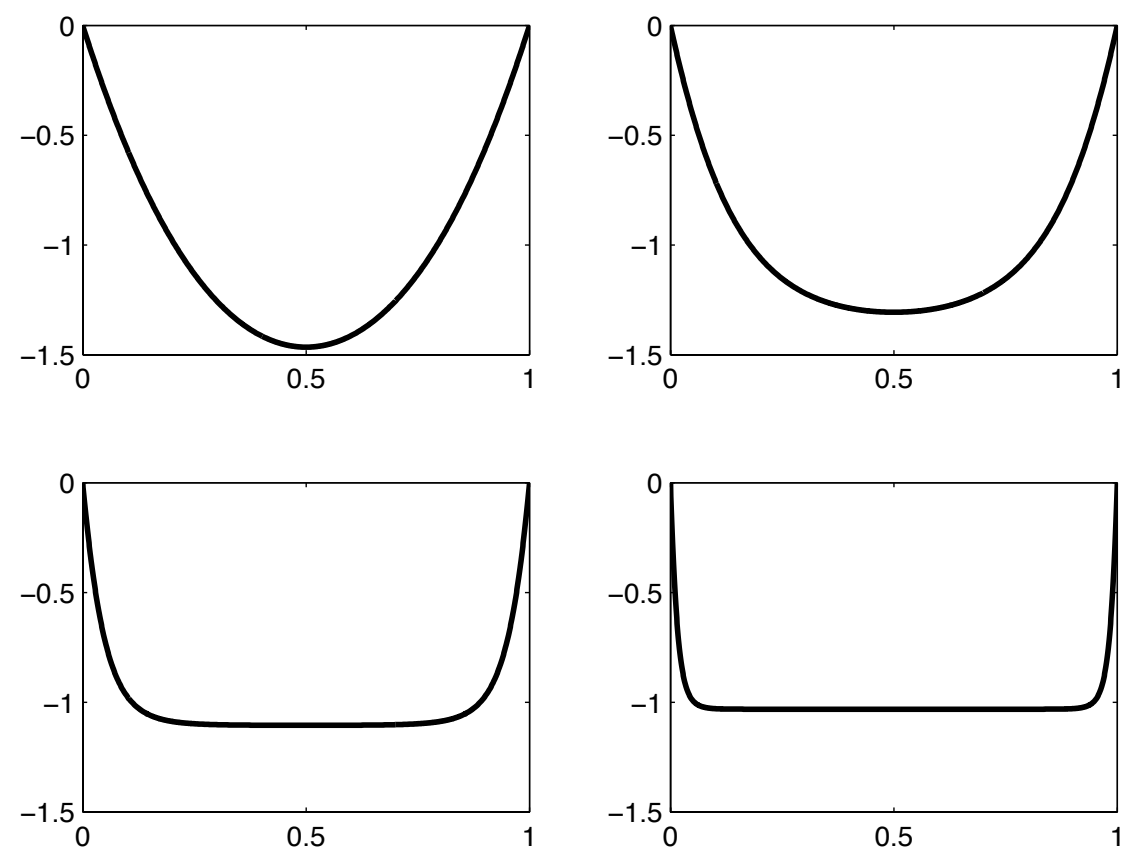

Fig. 6. The scaled radial velocity $s^{2} u(s, x)$ plotted against $x$ for four different values of $s$ with, from the upper left corner to the lower right corner, (a) $s=1$, (b) 0.1 , (c) 0.01 and (d) 0.001 .

In order sensibly to solve the PDE we need to start with the viscous solution (4.9) of Poiseuille flow valid for large $s$ and time-step the PDE towards lower values. We can then note that if $u>0$, that is a source flow, then (4.17) has the nature of a diffusion equation with $s$ in the role of time running backwards, and so is ill-posed. For this reason we restrict our attention now to a sink at the origin with $Q<0$ and the lower, negative sign taken in the boundary conditions (4.20) and elsewhere. The PDE was solved numerically using the routine d03pef from the NAG library, which integrates a system of first order PDEs. We start with the Poiseuille flow (4.9) at $s=s_{0}$ with $s_{0}=10$ and then step $s$ down until $s=0.001$. (We also start with the arbitrary choice $p\left(s_{0}\right)=-5$, and note that our calculations only determine $p$ up to the addition of a constant.)

Figures 6 and 7 show the scaled radial and cross components. Only for $s<0.1$ does the radial profile begin to differ appreciably from Poiseuille flow, and a cross flow component $v$ begins to develop. Note that the cross flow component $v$ remains small compared with $u$ as $s \rightarrow 0$ given the scalings set out in the figure captions. In this limit the radial profile $u$ clearly develops a form of a plug flow, with a constant mainstream velocity and thinning boundary layers where the no-slip condition is enforced. Similarly the cross flow develops boundary layers on each side of a linear profile. We will investigate this using 

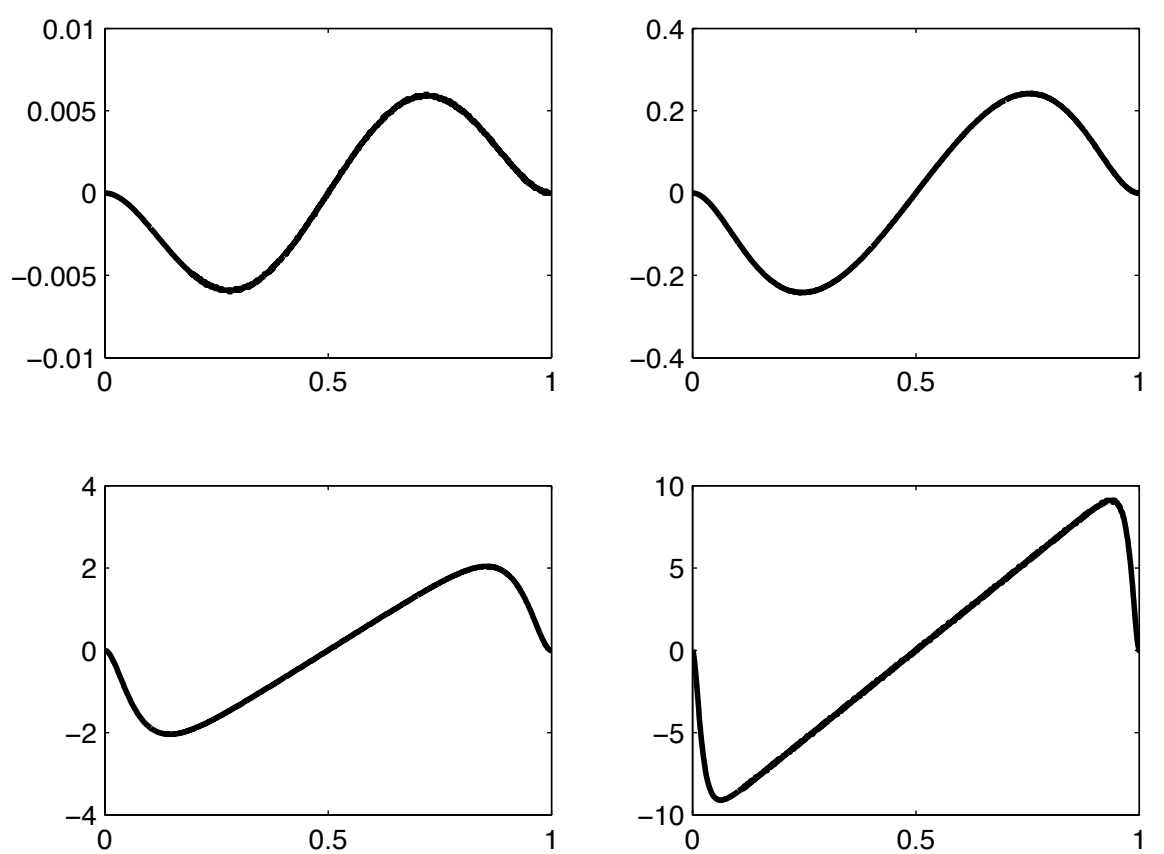

Fig. 7. The scaled cross velocity $s v(s, x)$ plotted against $x$ for four different values of $s$ with, from the upper left corner to the lower right corner, (a) $s=0.95$, (b) 0.095, (c) 0.0095 and (d) 0.00095 .

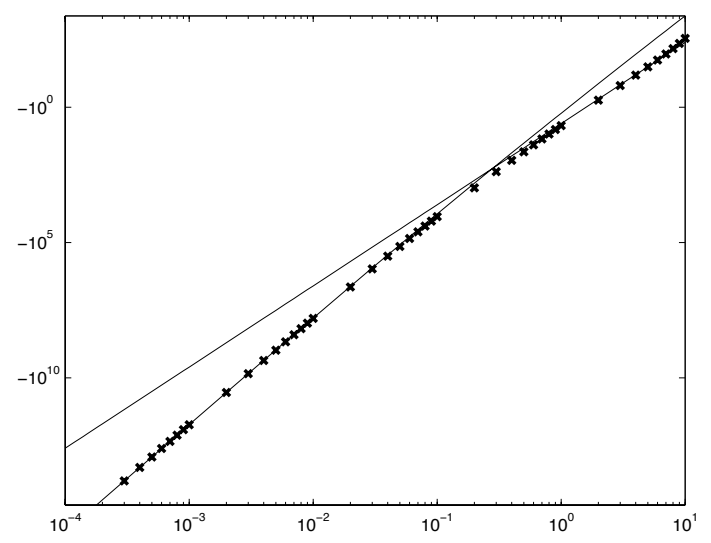

Fig. 8. The graph shows a log-log plot of the pressure magnitude $|p|$ (markers) against $s$, together with curves indicating the approximations $p=-\frac{1}{2} s^{-4}+P_{1} s^{-7 / 2}$ (with $P_{1}=a_{1}$ given in (4.35)), correct for small $s$, and $p=-4 s^{-3}$, correct for large $s$.

matched asymptotic expansions in the next section; here we only note that a careful examination of the data indicates a boundary layer width of order $\sqrt{s}$ in $x$ as $s \rightarrow 0$. 
In figure 8 the pressure magnitude $|p|$ has been plotted against $s$ in a log-log plot. This shows two power law regimes, one for large $s$ where $p \simeq-4 s^{-3}$ from the solution (4.9) in the viscous limit (rescaled according to (4.14) and (4.15)). However as $s$ decreases there is a transition to a dependence $p \simeq-\frac{1}{2} s^{-4}$, which we will confirm with our boundary layer theory, in (4.24) below.

\subsection{High Reynolds number limit}

In this section we set out the boundary layer theory for the flows that emerge in the limit $s \rightarrow 0$, as depicted in figures 6 and 7 . By symmetry we need only work on the range $0 \leq x \leq \frac{1}{2}$. We assume a boundary layer thickness of order $\sqrt{s}$ and plainly have a mainstream, outer region in which $\sqrt{s} \ll x \leq \frac{1}{2}$. In this latter region we pose an expansion

$$
\begin{aligned}
& u=s^{-2} U_{0}(x)+s^{-3 / 2} U_{1}(x)+\cdots, \quad v=s^{-3 / 2} V_{0}(x)+s^{-1} V_{1}(x)+\cdots, \\
& \psi=\Psi_{0}(x)+s^{1 / 2} \Psi_{1}(x)+\cdots, \quad p=s^{-4} P_{0}+s^{-7 / 2} P_{1}+\cdots .
\end{aligned}
$$

Note that the terms $P_{i}$ in the pressure are constants. The expansions for $u$, $v, \psi$ and $p$ are substituted into the governing PDEs (4.17) and (4.18). On equating powers of $s$ we rapidly obtain

$$
U_{0} \equiv a_{0}=-\left(-2 P_{0}\right)^{1 / 2}, \quad U_{1} \equiv a_{1}=P_{1}\left(-2 P_{0}\right)^{-1 / 2},
$$

where the $a_{i}$ are constants. In fact this simply represents an expansion of the exact mainstream solution $u(s, x)=-(-2 p(s))^{1 / 2}$ of plug flow, independent of $x$ (Ackerberg, 1965). The corresponding stream functions may be written as, from (4.18),

$$
\Psi_{0}=a_{0}\left(x-\frac{1}{2}\right)-\frac{1}{2}, \quad \Psi_{1}=a_{1}\left(x-\frac{1}{2}\right) .
$$

Here we have imposed the condition that $\psi=-\frac{1}{2}$ at the middle $x=\frac{1}{2}$ of the channel which results from (4.20) (lower sign) and symmetry in the $x$-range.

The other condition that $\psi=0$ on $x=0$ will be imposed not on the mainstream solution, but on the boundary layer. In fact, we anticipate that $a_{0}=-1$ as at leading order the mainstream carries all the flux. This will give $P_{0}=-\frac{1}{2}$ and so the leading order pressure as

$$
p \simeq-\frac{1}{2} s^{-4},
$$

in agreement with figure 8 . The leading order cross velocity is

$$
V_{0}=-\frac{1}{2} \Psi_{1}=-\frac{1}{2} a_{1}\left(x-\frac{1}{2}\right),
$$

from (4.18) and (4.21), and to determine this we need $a_{1}$. This requires the specification of the boundary layer, and we now turn to this. 


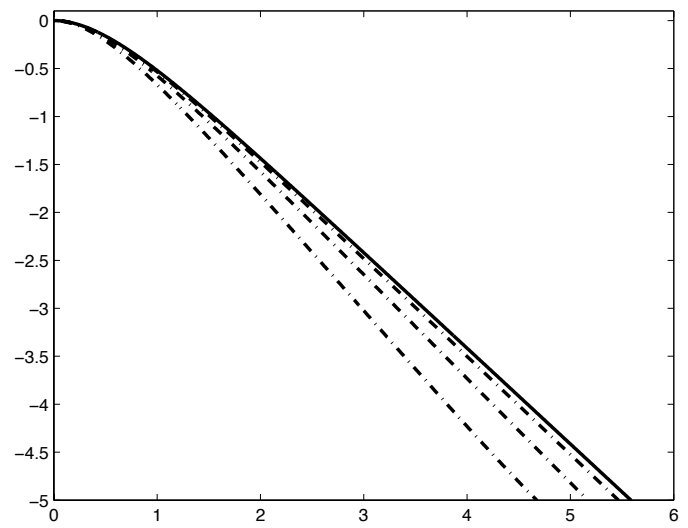

Fig. 9. Plotted is the stream function $\Psi_{0}(y)$ (solid) from the boundary layer ODE versus $y$. The corresponding quantity $s^{-1 / 2} \psi\left(s^{1 / 2} y, s\right)$ (dash-dot), obtained from the PDE, is also shown versus $y$ for $s=0.03,0.005$ and 0.0004 .

As the boundary layer thickness appears to be of order $\sqrt{s}$ we set

$$
x=s^{1 / 2} y,\left.\quad \frac{\partial}{\partial x}\right|_{s}=\left.\frac{1}{s^{1 / 2}} \frac{\partial}{\partial y}\right|_{s},\left.\quad \frac{\partial}{\partial s}\right|_{x}=\left.\frac{\partial}{\partial s}\right|_{y}-\left.\frac{y}{2 s} \frac{\partial}{\partial y}\right|_{s} .
$$

Without approximation the governing equations (4.17) and (4.18) then transform to

$$
\begin{gathered}
u\left(\frac{\partial}{\partial s}-\frac{y}{2 s} \frac{\partial}{\partial y}\right) u+\frac{v}{s^{3 / 2}} \frac{\partial u}{\partial y}=-\frac{\mathrm{d} p}{\mathrm{~d} s}+\frac{1}{s^{3}} \frac{\partial^{2} u}{\partial y^{2}} \\
u=\frac{1}{s^{5 / 2}} \frac{\partial \psi}{\partial y}, \quad v=-\frac{1}{s} \frac{\partial \psi}{\partial s}+\frac{y}{2 s^{2}} \frac{\partial \psi}{\partial y}
\end{gathered}
$$

We now expand (reusing the notation used for the mainstream),

$$
\begin{aligned}
u & =s^{-2} U_{0}(y)+s^{-3 / 2} U_{1}(y)+\cdots, \quad v=s^{-3 / 2} V_{0}(y)+s^{-1} V_{1}(y)+\cdots, \\
\psi & =s^{1 / 2} \Psi_{0}(y)+s \Psi_{1}(y)+\cdots, \quad p=s^{-4} P_{0}+s^{-7 / 2} P_{1}+\cdots .
\end{aligned}
$$

Substituting into the PDEs (4.27) and (4.28), and using $P_{0}=-\frac{1}{2}$ from the mainstream solution, gives an ODE at leading order,

$$
4 \Psi_{0}^{\prime} \Psi_{0}^{\prime}+\Psi_{0} \Psi_{0}^{\prime \prime}=4-2 \Psi_{0}^{\prime \prime \prime}
$$

This ODE is one of the general family discussed by Ackerberg (1965), and we note that it is different from that obtained by Vatistas \& Ghaly (1999) using their local linearisation procedure. This is solved with the no-slip condition and matching to linear behaviour in the mainstream,

$$
\Psi_{0}(0)=\Psi_{0}^{\prime}(0)=0, \quad \Psi_{0}^{\prime \prime} \rightarrow 0 \quad(y \rightarrow \infty) .
$$

The boundary value problem was solved numerically: the stream function $\Psi_{0}$ and flow components $U_{0}=\Psi_{0}^{\prime}$ and $V_{0}=\frac{1}{2}\left(-\Psi_{0}+y \Psi_{0}^{\prime}\right)$ for the solution are 


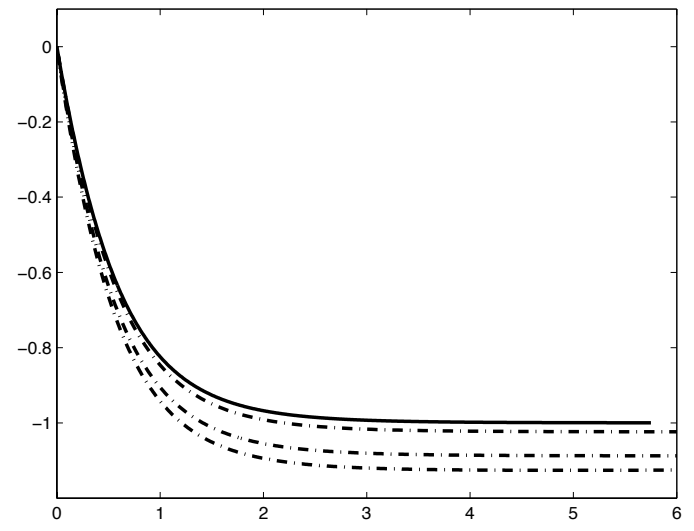

Fig. 10. Plotted is the radial velocity $U_{0}(y)$ (solid) from the boundary layer ODE versus $y$. The corresponding quantity $s^{2} u\left(s^{1 / 2} y, s\right)$ (dash-dot), obtained from the PDE, is also shown versus $y$ for $s=0.01,0.005$ and 0.0004 .

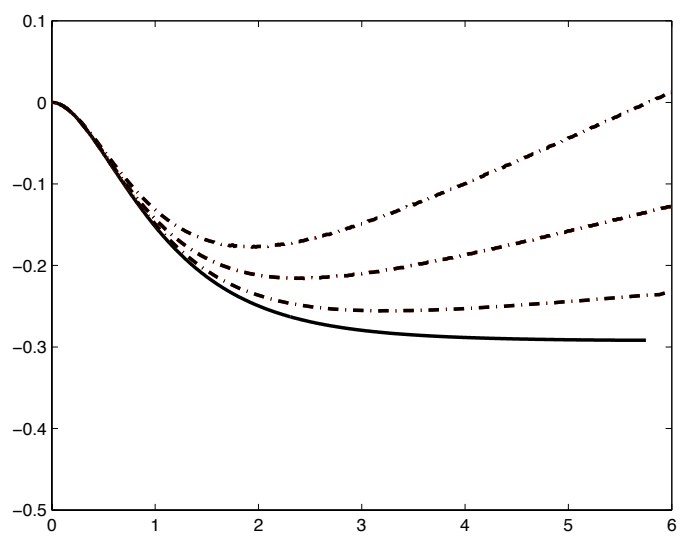

Fig. 11. Plotted is the cross velocity $V_{0}(y)$ (solid) from the boundary layer ODE versus $y$. The corresponding quantity $s^{3 / 2} v\left(s^{1 / 2} y, s\right)$ (dash-dot), obtained from the PDE, is also shown versus $y$ for $s=0.0025,0.00075$ and 0.00035 .

shown in figures 9, 10 and 11. Included in these figures are the solutions from the full PDE (see figures 6 and 7) plotted as a function of $y$ for several values of $s$ tending to zero. There is good agreement in the limit.

We also need the behaviour for large $y$, which is simply linear growth and takes the form,

$$
\Psi_{0} \sim-y+b_{0}, \quad V_{0} \sim-\frac{1}{2} b_{0}, \quad b_{0} \simeq 0.58 \quad(y \rightarrow \infty),
$$

with the value obtained numerically; see also figure 11 . We now compare the leading order stream function $\psi$ in the boundary layer, for $y \gg 1$,

$$
\psi^{\mathrm{bl}}=s^{1 / 2}\left(-y+b_{0}\right)+\cdots
$$


(from (4.29), (4.32)), with the mainstream solution for small $x=s^{1 / 2} y \ll 1$,

$$
\psi^{\mathrm{ms}}=a_{0}\left(s^{1 / 2} y-\frac{1}{2}\right)-\frac{1}{2}+s^{1 / 2} a_{1}\left(s^{1 / 2} y-\frac{1}{2}\right)+\cdots
$$

(from $(4.21),(4.23))$ we see that $a_{0}=-1$ as before, and

$$
a_{1}=-2 b_{0} \simeq-1.2
$$

This fixes the strength of the cross velocity (4.25), seen in figure 7 , and the next order correction $P_{1}$ to the pressure in (4.29) shown in figure 8.

\section{Flow in the parallel case for a narrow gap}

We now move to the parallel case, but omit much of the detail; for more information see Hall (2007). We cannot say much analytically about the apex region when $r^{\prime}=O(h)$ and we will avoid this region, taking only $r^{\prime} \gg h$. Recalling the definition of $\varepsilon$ and the Reynolds number in (2.6) and the basic non-dimensionalisation in (2.2), we can again define an effective Reynolds number as a function of radius. With inertial terms $\left(\mathbf{u}^{\prime} \cdot \nabla^{\prime}\right) \mathbf{u}^{\prime}$ scaling as $u^{\prime 2} / r^{\prime}$, the viscous terms $\nu \nabla^{\prime 2} \mathbf{u}^{\prime}$ as $\nu u^{\prime} / h^{2}$, and the flow magnitude $u^{\prime}=\mathcal{Q} / r^{\prime} h$, we may set

$$
\operatorname{Re}_{\mathrm{eff}}(r)=\left(u^{\prime 2} / r^{\prime}\right) /\left(\nu u^{\prime} / h^{2}\right)=h^{2} u^{\prime} / r^{\prime} \nu=h \mathcal{Q} / r^{\prime 2} \nu=\varepsilon r^{-2}
$$

Thus again with $\varepsilon \ll 1$ or $R e \gg 1$ we have a transition from viscous flow, for $r \gg \sqrt{\varepsilon}$ to flow with inertia at $r=O(\sqrt{\varepsilon})$ to high Reynolds number flow $r \ll \sqrt{\varepsilon}$ before reaching the apex region where $r=O(\varepsilon)$.

We use the biconical coordinates $(R, \phi, X)$, introduced by Ul'ev (2001), and depicted schematically in figure $1(\mathrm{~b})$,

$$
R=r \cos (\alpha-\theta), \quad X=r \sin (\alpha-\theta) .
$$

Here we restrict $X / R \leq \tan \alpha$ in general, and the layer is defined by the additional constraint that $0 \leq X \leq \varepsilon$ in the dimensionless framework. The line element is

$$
\mathrm{d} s^{2}=\mathrm{d} R^{2}+\zeta^{2} \mathrm{~d} \phi^{2}+\mathrm{d} X^{2}, \quad \zeta \equiv R \sin \alpha-X \cos \alpha .
$$

After some calculations, as in Batchelor (1967), the equations for momentum in the $R$ direction, $X$ direction and continuity, for axisymmetric flow, are 
obtained as

$$
\begin{aligned}
& u_{R} \frac{\partial u_{R}}{\partial R}+u_{X} \frac{\partial u_{R}}{\partial X}=-\frac{\partial p}{\partial R}+\frac{1}{\zeta} \frac{\partial}{\partial R}\left(\zeta \frac{\partial u_{R}}{\partial R}\right)+\frac{1}{\zeta} \frac{\partial}{\partial X}\left(\zeta \frac{\partial u_{R}}{\partial X}\right) \\
&-\frac{\sin ^{2} \alpha}{\zeta^{2}} u_{R}+\frac{\sin \alpha \cos \alpha}{\zeta^{2}} u_{X}, \\
& u_{R} \frac{\partial u_{X}}{\partial R}+u_{X} \frac{\partial u_{X}}{\partial X}=-\frac{\partial p}{\partial X}+\frac{1}{\zeta} \frac{\partial}{\partial R}\left(\zeta \frac{\partial u_{X}}{\partial R}\right)+\frac{1}{\zeta} \frac{\partial}{\partial X}\left(\zeta \frac{\partial u_{X}}{\partial X}\right) \\
&+\frac{\sin \alpha \cos \alpha}{\zeta^{2}} u_{R}-\frac{\cos ^{2} \alpha}{\zeta^{2}} u_{X}, \\
& \frac{1}{\zeta} \frac{\partial}{\partial R}\left(\zeta u_{r}\right)+\frac{1}{\zeta} \frac{\partial}{\partial X}\left(\zeta u_{X}\right)=0 .
\end{aligned}
$$

Continuity is satisfied using a stream function $\psi(R, X)$ with

$$
u_{R}=-\frac{1}{\zeta} \frac{\partial \psi}{\partial X}, \quad u_{X}=\frac{1}{\zeta} \frac{\partial \psi}{\partial R}
$$

and the boundary conditions are

$$
\psi(R, 0)=0, \quad \psi(R, \varepsilon)=\mp 1, \quad u_{R}(R, 0)=u_{R}(R, \varepsilon)=0 .
$$

Note that the upper sign in this last boundary condition refers to outflow $Q>0$ and the lower sign to inflow $Q<0$.

\subsection{Transition to high Reynolds number flow}

We can choose various possible scalings. Taking $R=O(1)$ and $X=\varepsilon Y$ with $0 \leq Y \leq 1$ gives a viscous regime, with a leading order solution,

$$
\begin{aligned}
u_{R} & \simeq \mp \frac{6}{\varepsilon R \sin \alpha} Y(Y-1), \quad u_{X} \simeq 0, \\
\psi & \simeq \pm Y^{2}(2 Y-3), \quad p \simeq \mp \frac{12}{\varepsilon^{3} \sin \alpha} \log R,
\end{aligned}
$$

analogous to that in (4.9), and corrections can be obtained from the effects of inertia and curvature (Hall, 2007). Instead of setting these out here, we move straight to the regime $R=O\left(\varepsilon^{1 / 2}\right)$ where inertia begins to become relevant as the effective Reynolds number increases to values of order unity.

We use length scales $Y$ and $S$ defined by

$$
X=\epsilon Y, \quad \frac{\partial}{\partial X}=\frac{1}{\epsilon} \frac{\partial}{\partial Y}, \quad R=\sqrt{\frac{\varepsilon}{\sin \alpha}} S, \quad \frac{\partial}{\partial R}=\sqrt{\frac{\sin \alpha}{\varepsilon}} \frac{\partial}{\partial S},
$$


and expand quantities as functions of $S$ and $X$ as

$$
\begin{aligned}
u_{R} & =\frac{1}{\sqrt{\sin \alpha}}\left(\varepsilon^{-3 / 2} u_{0}+\varepsilon^{-1} u_{1}+\cdots\right), \quad u_{X}=\varepsilon^{-1} v_{0}+\varepsilon^{-1 / 2} \varepsilon v_{1}+\cdots, \\
\psi & =\psi_{0}+\varepsilon^{1 / 2} \psi_{1}+\cdots, \quad p=\frac{1}{\sin \alpha}\left(\varepsilon^{-3} p_{0}+\varepsilon^{-5 / 2} p_{1}+\cdots\right) .
\end{aligned}
$$

As before we obtain that $p=p(S)$, independent of $Y$, and the following governing equations for $u_{0}, v_{0}, p_{0}$ and $\psi_{0}$, where we again drop the zero subscript,

$$
\begin{gathered}
u \frac{\partial u}{\partial S}+v \frac{\partial u}{\partial Y}=-\frac{\mathrm{d} p}{\mathrm{~d} S}+\frac{\partial^{2} u}{\partial Y^{2}} \\
u=-\frac{1}{S} \frac{\partial \psi}{\partial Y}, \quad v=\frac{1}{S} \frac{\partial \psi}{\partial S}
\end{gathered}
$$

We can obtain a single PDE for $\psi$,

$$
\frac{1}{S} \frac{\partial \psi}{\partial Y} \frac{\partial}{\partial S}\left(\frac{1}{S} \frac{\partial \psi}{\partial Y}\right)-\frac{1}{S^{2}} \frac{\partial \psi}{\partial S} \frac{\partial^{2} \psi}{\partial Y^{2}}=-\frac{\mathrm{d} p}{\mathrm{~d} S}-\frac{1}{S} \frac{\partial^{3} \psi}{\partial Y^{3}}
$$

and the boundary conditions are

$$
\psi(S, 0)=0, \quad \psi(S, 1)=\mp 1, \quad u(S, 0)=u(S, 1)=0 .
$$

We henceforth restrict to the case of inflow, $Q<0$, and the lower sign.

Note that equation (5.9) provides a solution valid in the limit of large $S$. We have used this to solve numerically for the fluid flow, stepping the variable $S$ from $S_{0}=10$ (with $p\left(S_{0}\right)=-5$ ) towards zero. Results are shown in figures 12 , 13 and 14, and we see a transition from a viscous, Poiseuille profile, to a plug flow bounded by thin layers. Note that these layers appear thinner than in the non-parallel case, figures 6 and 7, and in fact their width appears numerically to be of order $S$ as $S \rightarrow 0$.

\subsection{Analysis of plug flow and boundary layers}

We now set out the structure of the mainstream flow and boundary layers in the limit $S \rightarrow 0$ of high effective Reynolds number. The calculations parallel those for the non-parallel case, and so we just give the bare bones here. In the mainstream we pose an expansion,

$$
\begin{aligned}
& u=S^{-1} U_{0}(Y)+U_{1}(Y)+\cdots, \quad v=S^{-1} V_{0}(Y)+V_{1}(Y)+\cdots \\
& \psi=\Psi_{0}(Y)+S \Psi_{1}(Y)+\cdots, \quad p=S^{-2} P_{0}+S^{-1} P_{1}+\cdots .
\end{aligned}
$$

From the governing equations with $\psi=\frac{1}{2}$ at $Y=\frac{1}{2}$ we obtain

$$
U_{0} \equiv a_{0}=-\left(-2 P_{0}\right)^{1 / 2}, \quad U_{1} \equiv a_{1}=P_{1}\left(-2 P_{0}\right)^{-1 / 2}
$$



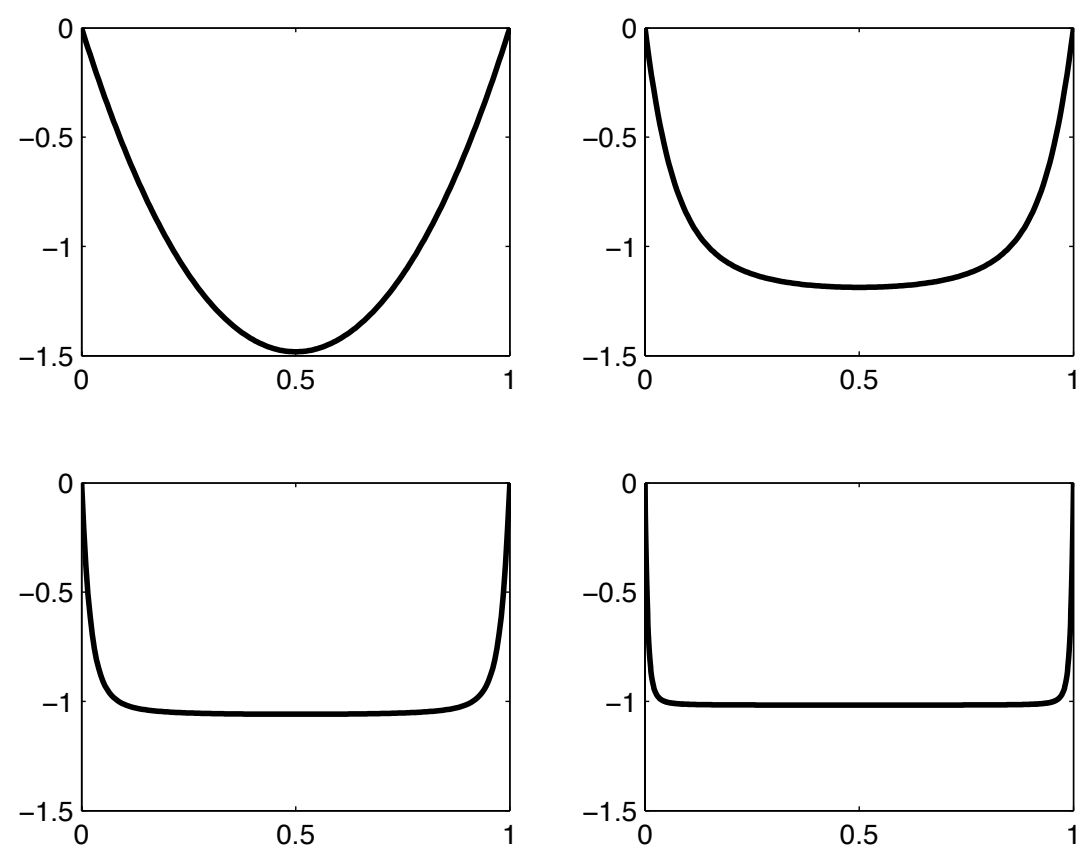

Fig. 12. The scaled radial velocity $S u(S, Y)$ plotted against $Y$ for four different values of $S$ with, from the upper left corner to the lower right corner, (a) $S=1$, (b) 0.1, (c) 0.03 and (d) 0.009 .

$$
\Psi_{0}=-a_{0}\left(Y-\frac{1}{2}\right)+\frac{1}{2}, \quad \Psi_{1}=-a_{1}\left(Y-\frac{1}{2}\right) .
$$

We anticipate that $a_{0}=-1$ from the boundary layer analysis and so

$$
P_{0}=-\frac{1}{2}, \quad V_{0}=\Psi_{1}=-a_{1}\left(Y-\frac{1}{2}\right) .
$$

To fix the constant $a_{1}$ and so the cross flow, we turn to the boundary layer.

The boundary layer is of thickness $S$ and so we set

$$
Y=S Z,\left.\quad \frac{\partial}{\partial Y}\right|_{S}=\left.\frac{1}{S} \frac{\partial}{\partial Z}\right|_{S},\left.\quad \frac{\partial}{\partial S}\right|_{Y}=\left.\frac{\partial}{\partial S}\right|_{Z}-\left.\frac{Z}{S} \frac{\partial}{\partial Z}\right|_{S},
$$

and the governing equations become

$$
\begin{gathered}
u\left(\frac{\partial}{\partial S}-\frac{Z}{S} \frac{\partial}{\partial Z}\right) u+\frac{v}{S} \frac{\partial u}{\partial Z}=-\frac{\mathrm{d} p}{\mathrm{~d} S}+\frac{1}{S^{2}} \frac{\partial^{2} u}{\partial Z^{2}}, \\
u=-\frac{1}{S^{2}} \frac{\partial \psi}{\partial Z}, \quad v=\frac{1}{S} \frac{\partial \psi}{\partial S}-\frac{Z}{S^{2}} \frac{\partial \psi}{\partial Z} .
\end{gathered}
$$

With the expansion,

$$
\begin{aligned}
& u=S^{-1} U_{0}(Z)+U_{1}(Z)+\cdots, \quad v=S^{-1} V_{0}(Z)+V_{1}(Z)+\cdots \\
& \psi=S \Psi_{0}(Z)+S^{2} \Psi_{1}(Z)+\cdots, \quad p=S^{-2} P_{0}+S^{-1} P_{1}+\cdots
\end{aligned}
$$



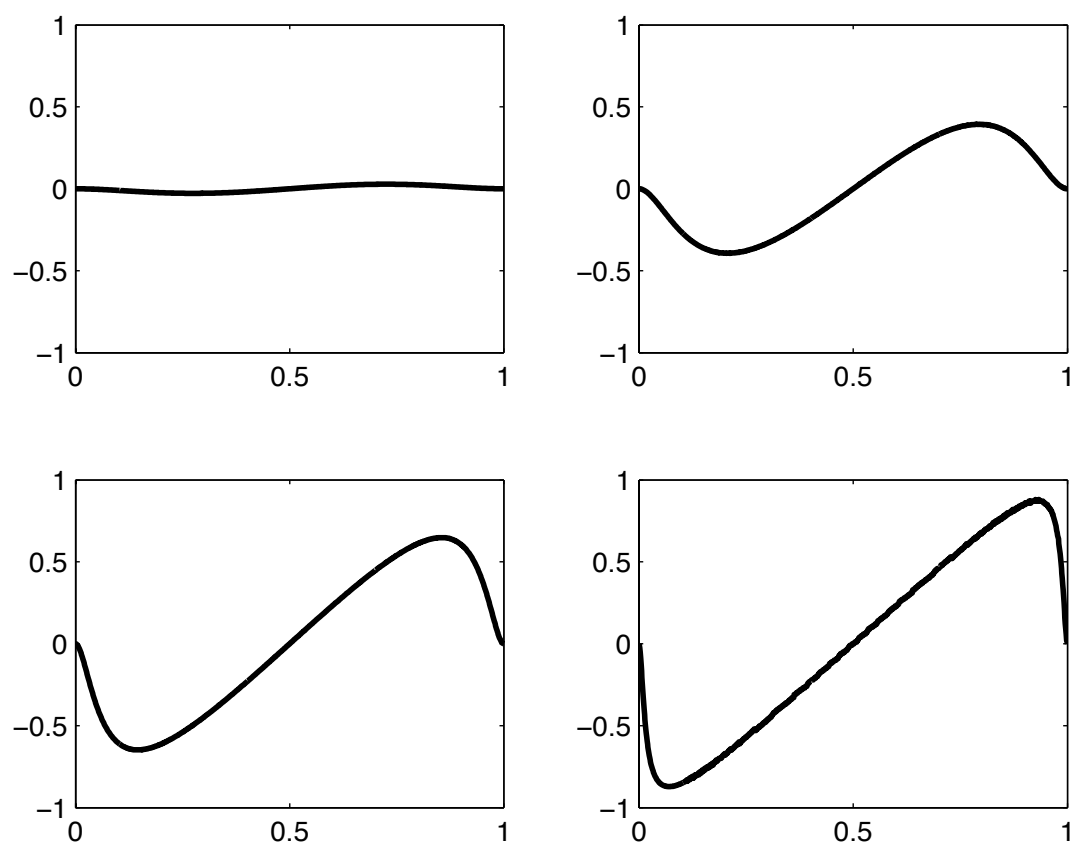

Fig. 13. The scaled cross velocity $S v(S, Y)$ plotted against $Y$ for four different values of $S$ with, from the upper left corner to the lower right corner, (a) $S=0.45$, (b) 0.085 , (c) 0.033 and (d) 0.0085 .

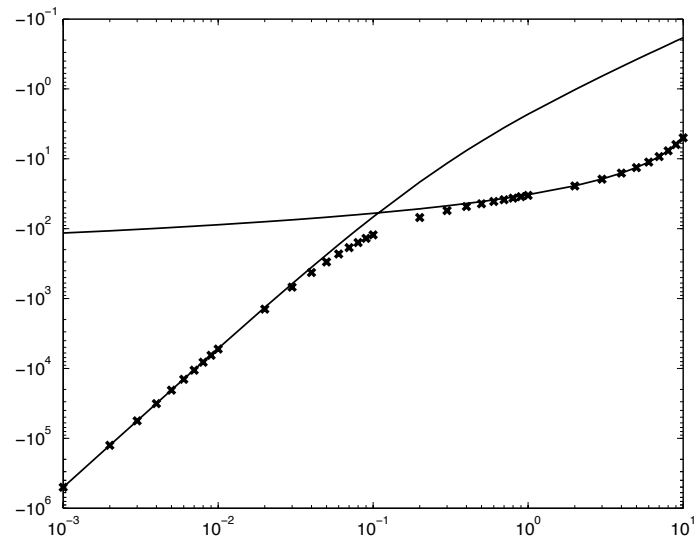

Fig. 14. The graph shows a log-log plot of the pressure magnitude $|p|$ (markers) against $S$, together with lines indicating the approximations $p=-\frac{1}{2} S^{-2}+P_{1} S^{-3}$ (with $P_{1}=a_{1}$ given in (5.27)), correct for small $S$, and $p=12 \log (S / 10)-5$, correct for large $S$.

and $P_{0}=-\frac{1}{2}$ we obtain the following ODE at leading order,

$$
\Psi_{0}^{\prime} \Psi_{0}^{\prime}+\Psi_{0} \Psi_{0}^{\prime \prime}=1+\Psi_{0}^{\prime \prime \prime}
$$




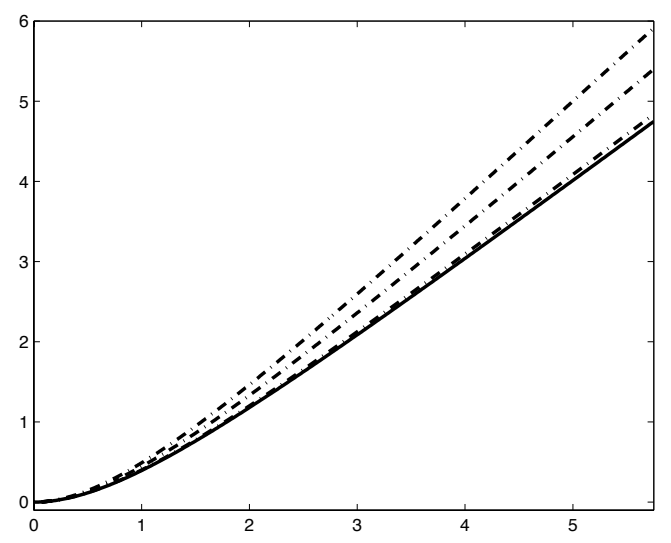

Fig. 15. Plotted is the stream function $\Psi_{0}(Z)$ (solid) from the boundary layer ODE versus $Z$. The corresponding quantity $S^{-1} \Psi(S Z, S)$ (dash-dot), obtained from the PDE, is also shown versus $Z$ for $S=0.1,0.06$ and 0.01 .

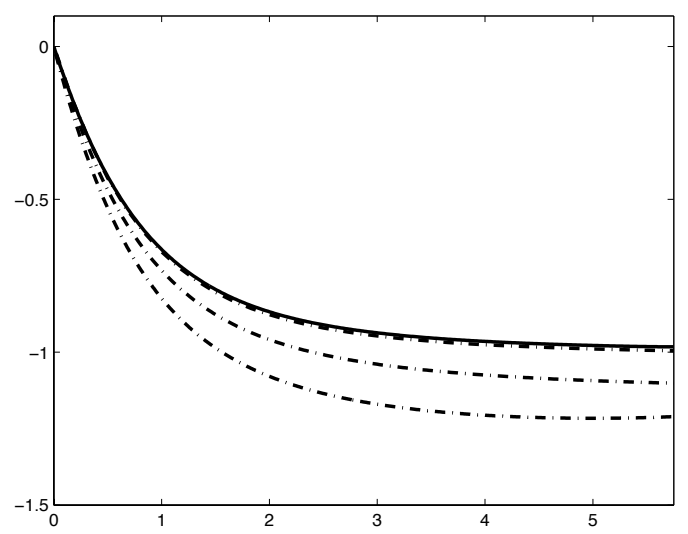

Fig. 16. Plotted is the radial velocity $U_{0}(Z)$ (solid) from the boundary layer ODE versus $Z$. The corresponding quantity $S u(S Z, S)$ (dash-dot), obtained from the PDE, is also shown versus $Z$ for $S=0.1,0.05$ and 0.006 .

with the boundary conditions

$$
\Psi_{0}(0)=\Psi_{0}^{\prime}(0)=0, \quad \Psi_{0}^{\prime \prime} \rightarrow 0 \quad(Z \rightarrow \infty) .
$$

This boundary value problem was solved numerically: the stream function and flow components $U_{0}=-\Psi_{0}^{\prime}$ and $V_{0}=\Psi_{0}-Z \Psi_{0}^{\prime}$ for the solution are shown in figures 15, 16 and 17, together with the data from the full PDE, as in figures 12 and 13, with good agreement as $S \rightarrow 0$. Finally, for large $y$, we obtain from the numerical solution that

$$
\Psi_{0} \sim Z+b_{0}, \quad V_{0} \sim b_{0}, \quad b_{0} \simeq-0.90 \quad(Z \rightarrow \infty) .
$$




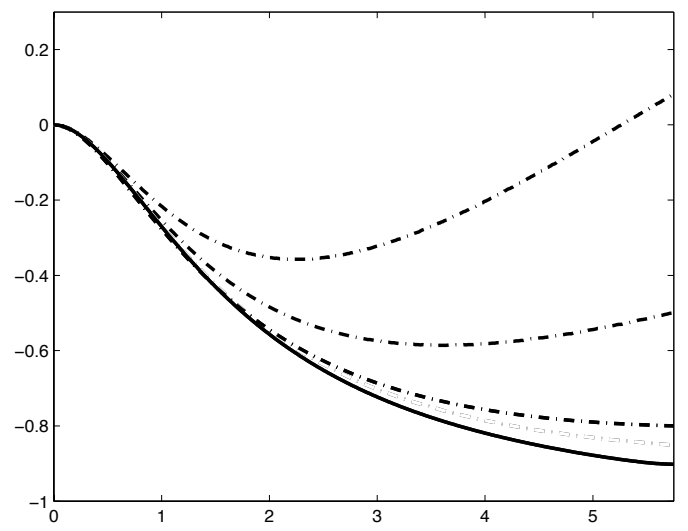

Fig. 17. Plotted is the cross velocity $V_{0}(Z)$ (solid) from in the boundary layer ODE versus $Z$. The corresponding quantity $S v(S Z, S)$ (dash-dot), obtained from the $\mathrm{PDE}$, is also shown versus $Z$ for $S=0.095,0.045$ and 0.015 .

Matching the boundary layer solution and the mainstream confirms that $a_{0}=$ -1 as assumed, and fixes

$$
a_{1}=2 b_{0} \simeq-1.8
$$

which determines the strength of the cross velocity (5.19), seen in figure 13 .

\section{Discussion}

We have discussed converging flow between two cones in the Stokes regime, for any cone opening, and found singular behaviour for certain wide, asymmetric openings, which had not been noted by previous authors. Moving on to flows with inertia, we studied converging flows, and obtained both PDEs describing the transition from Stokes flow to high Reynolds number flow, and ODEs describing the structure of the boundary layers that emerge. These governing equations for the non-parallel case were also obtained by Ackerberg (1965) but the PDE was not solved numerically. Our studies are limited to the case of a narrow angular gap for the non-parallel geometry and assumed a narrow gap, or equivalently large Reynolds number, for the parallel geometry. These two cases are broadly similar, but the scalings and boundary layer structure are different; for example the boundary layers thin more rapidly in the parallel case. In each case plug flows emerge, as have been seen in previous studies.

There are a number of ways in which the present studies could be developed. It would be interesting to consider the case of the non-parallel geometry with a general opening, rather than a narrow angular gap. This would make contact with the results for Stokes flow which indicate a breakdown for asymmetric, wide openings, and results which suggest that weak inertial effects can also 
lead to breakdown in the symmetric case (Fohr \& Mallet, 1975; Renaud, 1980). We make some tentative steps in an appendix: for a general opening in the non-parallel geometry, there is no small parameter to allow the derivation of a PDE that is significantly simpler than the full Navier-Stokes system. However if we assume that a plug flow emerges as $r \rightarrow 0$ then we can say something about its structure. We have also focussed in the present paper on converging flows, as this gave a well-posed PDE, but the case of diverging flows needs to be better understood.

Other physical effects can be brought in, in particular the effect of the rotation of cones, as in Troshkin (1973); we note that the effective Reynolds number for this effect increases with radius, unlike the flows we have discussed in the present paper. With convergence and rotation, a link may be made to classical stability problems involving Ekman instability and Couette-Poiseuille flows, not to mention Taylor vortices (Hoffman et al., 1998; Hoffman \& Busse, 1999, 2001; Ponty et al., 2003). Finally there is the question of the stability of the plug flows and boundary layers whose structure we have determined in our study.

\section{Acknowledgements}

We are grateful to the EPSRC which supports OH under grant GR/T08968/01. We thank Prof. Andrew Soward for useful discussions.

\section{A Appendix: Plug flow for wide cone openings}

We sketch the asymptotics of a plug flow in the limit $r \rightarrow 0$ for a wide cone opening in the non-parallel case, that is for general values of $\alpha$ and $\beta$ with $0<\alpha<\beta<\pi$. Starting with the general equations (3.1), (4.1) and (4.2), we expand in the mainstream

$$
\begin{gathered}
u_{r}=r^{-2} U_{0}(\theta)+r^{-3 / 2} U_{1}(\theta)+\cdots, \quad v_{\theta}=r^{-3 / 2} V_{0}(\theta)+r^{-1} V_{1}(\theta)+\cdots \\
\psi=\Psi_{0}(\theta)+r^{1 / 2} \Psi_{1}(\theta)+\cdots, \quad p=r^{-4} P_{0}(\theta)+r^{-7 / 2} P_{1}(\theta)+\cdots
\end{gathered}
$$

Substituting into the governing equations rapidly gives us that at leading order $P_{0}$ is a constant and that

$$
U_{0} \equiv a_{0}=-\left(-2 P_{0}\right)^{1 / 2}
$$


is also constant. Anticipating the boundary conditions for inflow on the leading order stream function, $\Psi_{0}=0$ at $\theta=\alpha$ and $\Psi_{0}=-1$ at $\theta=\beta$, gives

$$
\begin{gathered}
\Psi_{0}=-\frac{\cos \alpha-\cos \theta}{\cos \alpha-\cos \beta}, \\
a_{0}=-\frac{1}{\cos \alpha-\cos \beta}, \quad P_{0}=-\frac{1}{2(\cos \alpha-\cos \beta)^{2}} .
\end{gathered}
$$

At the next order, we do not generally have constant $P_{1}$ but instead we obtain

$$
U_{0} U_{1}=-P_{1}, \quad U_{0} V_{0}=2 P_{1}^{\prime}
$$

from the momentum equations and

$$
\sin \theta U_{1}=\Psi_{1}^{\prime}, \quad-2 \sin \theta V_{0}=\Psi_{1}
$$

from continuity. Putting in $U_{0}=a_{0}$, a constant, gives a linear system which can be written as

$$
\Psi_{1}=4 \sin \theta \frac{\mathrm{d}}{\mathrm{d} \theta}\left(\frac{1}{\sin \theta} \frac{\mathrm{d} \Psi_{1}}{\mathrm{~d} \theta}\right)
$$

and can be solved in terms of hypergeometric functions. This introduces two unknown constants into the problem.

Focussing on the boundary layer at $\theta=\alpha$, we set $\kappa=\left(-2 P_{0}\right)^{1 / 4}$, a constant,

$$
\theta=\alpha+\kappa^{-1} r^{1 / 2} y,\left.\quad \frac{\partial}{\partial \theta}\right|_{r}=\left.\frac{\kappa}{r^{1 / 2}} \frac{\partial}{\partial y}\right|_{r},\left.\quad \frac{\partial}{\partial r}\right|_{\theta}=\left.\frac{\partial}{\partial r}\right|_{y}-\left.\frac{y}{2 r} \frac{\partial}{\partial y}\right|_{r},
$$

and expand quantities as

$$
\begin{aligned}
& u=\kappa^{2} r^{-2} U_{0}(y)+\cdots, \quad v=\kappa r^{-3 / 2} V_{0}(y)+\cdots, \\
& \psi=\kappa \sin \alpha r^{1 / 2} \Psi_{0}(y)+\cdots, \quad p=r^{-4} P_{0}+\cdots .
\end{aligned}
$$

This scaling yields the ODE (4.30) we had before, for which $V_{0}$ tends to the value given in (4.32) given above as $y \rightarrow \infty$. This and a similar equation for the other boundary layer fix the unknown constants in the main-stream cross flow given by $\Psi_{1}$; see (A.6) and (A.7).

\section{References}

Abramowitz, M. \& Stegun, I.A. 1965 Handbook of mathematical functions. Dover.

Ackerberg, R. C. 1965 The viscous incompressible flow in a cone. J. Fluid Mech. 21, 47-81. 
Bark, F.H., Johansson, A.V. \& Carlsson, C.-G. 1984 Axisymmetric stratified two-layer flow in a rotating conical channel. J. Méc. Th. Appl. 3, 861-878.

Batchelor, G.K. 1967 An introduction to fluid dynamics. Cambridge University Press.

Bond, W.N. 1927 Theory of liquid flow through cones. Proc. Phys. Soc. 40, $1-6$.

Brown, S.N. \& Stewartson, K. 1965 On similarity solutions of the boundarylayer equations with algebraic decay. J. Fluid Mech. 23, 673-687.

Fohr, J.P. \& Mallet, J. 1975 Ecoulement visquex entre deux cones coaxiaux. Appl. Sci. Res. 30, 221-235.

Fraenkel, L.E. 1962 Laminar flow in symmetrical channels with slightly curved walls. I. on the Jeffrey-Hamel solutions for flow between plane walls. Proc. Roy. Soc. A 267, 119-138.

Hall, O. 2007 Fluid flow between concentric cones. PhD Thesis, University of Exeter.

Hall, O., Hills, C.P. \& Gilbert, A.D 2007 Slow flow between concentric cones. QJMAM 60, 27-48

Hewitt, R.E. 2004 Toroidal eddies in slowly swirling flows. Unpublished note.

Hoffmann, N.P. \& Busse, F.H. 1999 Instabilities of shear flows between two coaxial differentially rotating cones. Phys. Fluids 11, 1676-1678.

Hoffmann, N.P. \& Busse, F.H. 2001 Linear instability of Poiseuille-CouetteEkman flows: local results for flows between differentially rotating disks with throughflow. Phys. Fluids 13, 2735-2738.

Hoffmann, N.P., Busse, F.H. \& Chen, W.-L. 2001 Transition to complex flows in the Ekman-Couette layer. J. Fluid Mech. 366, 311-331.

Hynes, T.P. 1978 Stability of thin films, PhD thesis. University of Cambridge.

Malhotra, C.P., Weidman, P.D. \& Davies, A.M.J 2005 Nested toroidal vortices between concentric cones. J. Fluid Mech. 522, 117-139.

Malyuga, V.S. 2005, Viscous eddies in a circular cone. J. Fluid Mech. 522, $101-116$.

Moffatt, H.K. 1980 The asymptotic behaviour of solutions of the Navier-Stokes equations near sharp corners. In Approximation methods for Navier-Stokes problems, ed. R. Rautmann. Springer-Verlag.

Moffatt, H.K. \& Duffy, B.R. 1980 Local similarity solutions and their limitations, J. Fluid Mech. 96, 299-313

Nakamura, S., Masuzawa, T. \& Yamane, T. 2001 Unsteady flows in a thin cavity between rotating and non-rotating cones. In 2001 Proceedings of ASME Fluids Engineering Division Summer Meeting, Volume 1, pp. 1-8.

Noui-Mehidi, M.N., Salem, A., Legentilhomme, P. \& Legrand, J. 1999 Apex angle effects on the swirling flow between cones induced by means of a tangential inlet. Int. J. Heat \& Fluid Flow 20, 405-413.

Noui-Mehidi, M.N., Ohmura, N. \& Kataoka, K. 2002 Mechanism of mode selection for Taylor vortex flow between coaxial conical rotating cylinders. J. Fluids Struct. 16, 247-262.

Ohmura, N., Noui-Mehidi, M.N., Sasaki, K., Kitajima, K. \& Kataoka, K. 
2004 Mixing characteristics in a conical Taylor-Couette flow system at low Reynolds numbers. J. Chem. Eng. Japan 37, 546-550.

Ponty, Y., Gilbert, A.D. \& Soward, A.M. 2003 The onset of thermal convection in Ekman-Couette shear flow with oblique rotation. J. Fluid Mech. 487, 91-123.

Renaud, M.F. 1980 Écoulement divergent d'un fluide visqueux incompressible entre deux cônes de même axe et de même sommet. J. de Méc. 19, 415-431.

Shankar, P.N. 2005 Moffatt eddies in the cone. J. Fluid Mech. 539, 113-135.

Stow, S.R., Duck, P.W. \& Hewitt, R.E. 2001, Three-dimensional extensions to Jeffery-Hamel flow. Fluid Dyn. Res. 29, 25-46.

Troshkin, O.A. 1973 Calculations of hydrodynamical characteristics of viscous fluid flow between rotating cones. Teor. Osnovy Khim. Tekhnol. 7, 897-903.

Ul'ev L.M. 2001 Laminar heat transfer in a liquid flowing in a diverging conical annular channel with a varied inner-wall temperature. Theoretical Foundations Chem. Eng. 35, 28-38.

Vatistas, G.H. \& Ghaly, W.S. 1999 Converging and diverging flow in narrow conical passages. Acta Mech. 136, 209-222.

Wimmer, M. 1995 An experimental investigation of Taylor vortex flow between conical cylinders. J. Fluid Mech. 292, 205-227. 